\title{
Determination of the Skip-Stop Scheduling for a Congested Transit Line by Bilevel Genetic Algorithm
}

\author{
Huimin Niu* \\ School of Traffic and Transportation, Lanzhou Jiaotong University, \\ Lanzhou 730070, PR China \\ Received 11 July 2011 \\ Accepted 25 November 2011
}

\begin{abstract}
This study focuses on how to determine the skip-stop scheduling for a congested urban transit line during the morning rush hours. A transit schedule with uneven headways and skip-stop operations is adopted to match the time-dependent demands and accelerate the circulative utilizations of vehicles. This schedule allows the buses to run on unequal vehicle-departure intervals and to skip some stations. A nonlinear programming model is formulated to minimize the overall waiting times and the in-vehicle crowded costs subjected to a limited number of vehicles. According to the layered characteristics of the skip-stop scheduling, a bilevel genetic algorithm is developed to solve the proposed model. The possible departure times of vehicles at the terminal are searched by the outer genetic algorithm and the skip-stop operations are solved by the inner genetic algorithm. Finally, the proposed model and algorithm are successfully tested with the help of a real-world case.
\end{abstract}

Keywords: Transit scheduling, Skip-stop operation, Optimization, Bilevel genetic algorithm.

\section{Introduction}

The design of the transit scheduling for a public bus line, as a key component of the urban transit operation and management, is in fact how to determine the arrival and departure time for each bus at each station on the transit line according to the characteristics of the passenger demands. The spatial and temporal imbalance of the transit demand distribution represents the congestions merely occurring at some stations or at some times. Such demand patterns cannot be effectively satisfied by a regular transit schedule. An irregular scheduling which allows the buses to run on uneven headways and skip some stations can accommodate the timedependent passenger demands and accelerate the circulative utilizations of vehicles. Such an improved scheduling also evidently reduces the passenger waiting times and improves the transit service quality provided by a limited number of vehicles.

The transit scheduling problem has received much attention in the past years. ${ }^{1-4}$ The passenger satisfaction on bus services has a high correlation with the attributes of the services supplied. ${ }^{5}$ As for the passenger demands with varying and in comparison with fixed frequencies, the headway in a transit-route system is constructed by Banks. ${ }^{6}$ It is revealed that the total fleet size required for the operation of a bus network with the mixed-size is smaller than the need for the operation with the samescale regular size. ${ }^{7}$ The fleet size required for a bus line is decreased by inserting short-turn trips taking the headways as inputs. ${ }^{8}$ A particular service pattern including not only the short-turn strategy but also vehicle size as well as frequencies should be required to

* Corresponding author: hmniu@mail.lzjtu.cn 
supply the service over different operation periods. ${ }^{9} \mathrm{By}$ skipping a number of stops in high-frequency transit operations, the deadheading strategy could shorten the residence time of passengers in the bus transport system. ${ }^{10}$ The real-time scheduling strategy aiming to strike an optimal balance between the benefits of operators and passengers is formulated by Fu et al. ${ }^{11}$ An optimization method is proposed in Ref. 12 for designing limited-stop services which minimize both the travel time of passengers and the operating cost of an urban bus corridor by optimizing the transport services with different arriving frequencies of various types of buses.

The existing studies have paid much attention to the regular schedules and yet failed to explore the problem of transit scheduling with uneven vehicle-departure intervals based on time-dependent passenger demands. Moreover, the irregular transit schedules integrated with special operation activities in a unified framework has not yet been adequately considered previously. This research focuses on how to determine a transit schedule for a congested public bus line in morning peak hours. A hybrid procedure embedded in bilevel genetic algorithm is developed to solve the model due to its large-scale and complicated characteristics.

The remainder of the paper is organized as follows. Section 2 analyzes the transit operation process and passenger behavior in some assumptions. In Section 3, an optimization model of transit scheduling with skipstop operation for a congested public bus line is established. A bilevel genetic algorithm is developed to solve the optimization model in Section 4. In Section 5, a numerical example is provided to illustrate the application of the proposed model and algorithm. The last section draws conclusions and proposes some future research issues.

\section{Problem Statement}

This study considers a ring-like urban public transit line with $N$ stations which are sequentially numbered as $1,2, \cdots, N$. Station 1 and Station $N$ denote the identical terminal as shown in Fig.1. Each bus departs from station 1 and then makes a loop-movement to come back to station $N$ for a new departure.

To accelerate the circulative utilizations of a limited number of vehicles, a transit schedule with the skip-stop operation which allows the buses to skip some of the stations is considered in this study. For example, bus 1 skips station 3 and station 5 and bus 2 skips station 2 and station 4, as shown in Fig. 2.

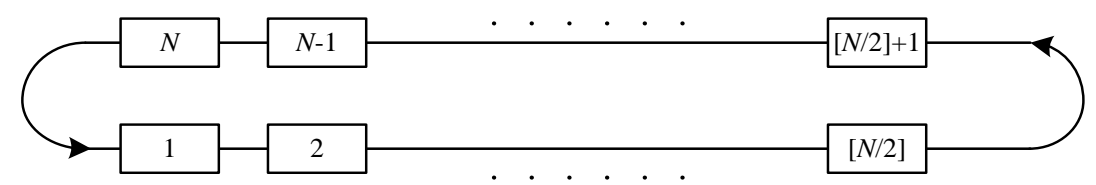

Fig. 1 The structure illustration of a ring-like urban public transit line

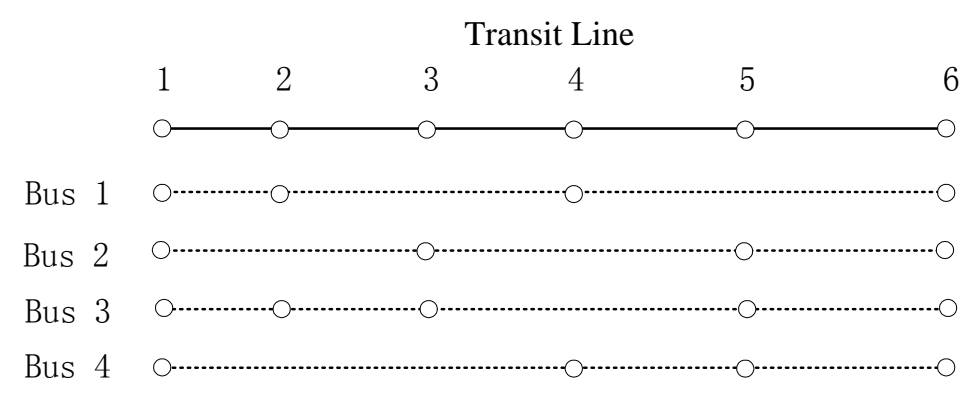

Fig. 2 A transit schedule with skip-stop operation

Let $u$ and $v$ index the stations, and $j$ index the buses. Recurring to the time-space trajectory applied in some references $^{2,11}$, this study uses Fig. 3 to illustrate the traversing process of buses at a station. There are four 
consecutive buses, i.e. $j, j+1, j+2$ and $j+3$, where bus $j, j+1$ and $j+3$ stop at station $u$, while bus $j+2$ skips at the station. In Fig. 3, two nodes are used to denote the arrival and departure of a bus respectively if the vehicle stays at the station, and one node is used to represent the skip process. Let $A_{j}^{u}$ and $D_{j}^{u}$ respectively indicate the arrival and departure time of the bus $j$ which stops at sation $u$. What needs to point out is that $A_{j}^{u}$ and $D_{j}^{u}$ are equal if bus $j$ skips at station $u$.

Let $t$ index the times. To consider the time-dependent demand matrix along the public bus line, the number of passengers who arrive at station $u$ at time $t$ toward station $v$ is denoted as $P^{u, v}(t)$. In fact the vehicles have to stop at the terminal and some line-joint stations. At the other stations, however, the vehicles may stop or skip. Therefore, this study uses $\Phi_{1}$ and $\Phi_{2}$ to interpret the sets of sure-stop and choice-stop stations respectively.

For a given station as shown in Fig. 3, the arrival process of passengers is separated as a sequence of consecutive intervals by the partition nodes whose time stamps correspond to the bus departure times. During each interval, a waiting passenger could board the departure bus if it also stops at his/her destination station. For example, a passenger associated with $P^{u, v}(t)=1$ and $t \in\left(D_{j}^{u}, D_{j+1}^{u}\right]$ can board bus $j+1$ if the vehicle stops at the destination station of this passenger. However, the effective loading period for bus $j+3$ should be extended $\left(D_{j+1}^{u}, D_{j+3}^{u}\right]$ as bus $j+2$ skips at station $u$.

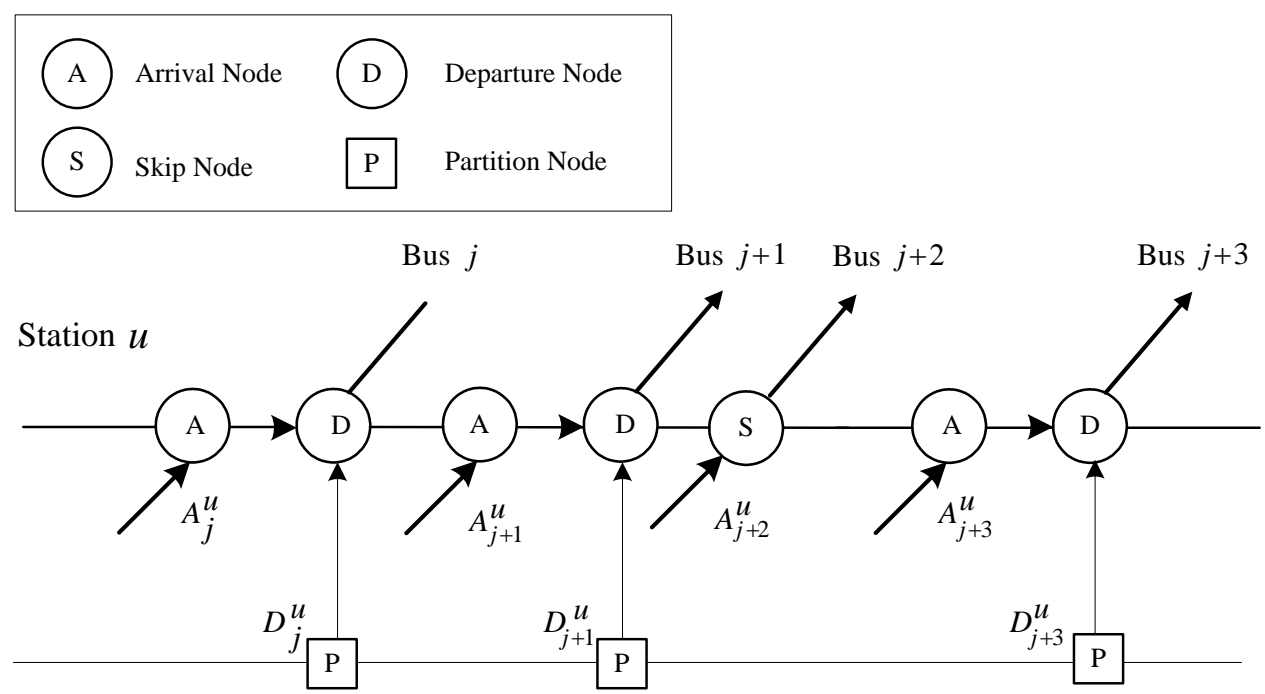

Fig.3 Illustration of transit operation process at a station

\section{Model}

\subsection{Constraints}

The notation and parameters used in this paper are listed as follows.

Index

$u, v$ index of station

$j \quad$ index of bus $t$ index of time

Input

$C$ the bus capacity, i.e. the maximum number of passengers able to be accommodated by a vehicle

$m$ the number of supplied vehicles

$s^{u}$ the dwell time at station $u$

$r^{u}$ the running time from station $u$ to station $u+1$

$P^{u, v}(t)$ the number of passengers who arrive at station $u$ at time $t$ toward station $v$ 


\section{Decision variables}

$A_{j}^{u} \quad$ arrival time of bus $j$ which stops at station $u$

$D_{j}^{u} \quad$ departure time of bus $j$ which stops at station $u$

$Y_{j}^{u} \quad$ binary variables to indicate the skip-stop operation, which is equal to 1 if bus $j$ stops at station $u$ and 0 otherwise

\section{Dependent variables}

$X_{j}^{u}$ the number of remaining passengers in bus $j$ after the bus departs from station $u$

$I_{j}^{u} \quad$ the number of boarded passengers in bus $j$ during the stay of the bus at station $u$

$O_{j}^{u}$ the number of alighted passengers in bus $j$ after its arrival at station $u$

$K$ the total number of buses departing from the terminal over the study period

$T_{j}$ the required time of bus $j$ for taking a ring-like circulation, which consists of the running time at the sections, the dwell time at the stations and the recovery time at the terminal.

It is noted that the dependent variables can be determined recursively by the model and the associated decision variables. Moreover, decision variables $A_{j}^{u}$ and $D_{j}^{u}$ are entirely decided by the departure times of vehicles at the terminal $D_{j}^{1}$. As a result, the independent decision variables in this study are actually $D_{j}^{1}$ and $Y_{j}^{u}$. We also notice that the value of $Y_{j}^{u}$ can only take 1 if $u \in \Phi_{1}$ and $Y_{j}^{u}$ can take 1 or 0 arbitrarily if $u \in \Phi_{2}$. Let $\delta_{j}^{u, v}$ indicate bus $j$ whether it stops both at station $u$ and station $v$ or not. It is equal to 1 if the bus stops at these two stations simultaneously and 0 otherwise. For the stopping bus $j$ at station $u$, let $F(j)$ index the latest bus which is prior to bus $j$ and also stops at station $u$, namely $Y_{j}^{u}=1$ and $Y_{F(j)}^{u}=1$. It can be calculated as follows.

$$
F(j)=\max \left\{j^{\prime} \mid j^{\prime}<j, Y_{j^{\prime}}^{u} \times Y_{j}^{u}=1\right\}
$$

Then, the numbers of passengers boarding and alighting at station $u$ for bus $j$ are explained below.

$$
\begin{aligned}
& \sum_{v=u+1}^{N} \int_{t \in\left(D_{F(j)}^{u}, D_{j}^{u}\right]} \delta_{j}^{u, v} \times P^{u, v}(t) d t \\
& \sum_{v=1}^{u-1} \int_{t \in\left(D_{F(j)}^{v}, D_{j}^{v}\right]} \delta_{j}^{v, u} \times P^{v, u}(t) d t
\end{aligned}
$$

Based on the above analyses, the constraints associated with the skip-stop scheduling problem for a congested public bus line is formulated as follows.

$$
\begin{gathered}
\delta_{j}^{u, v}=Y_{j}^{u} \times Y_{j}^{v} \\
A_{j}^{u}=D_{j}^{u-1}+r^{u-1} \\
D_{j}^{u}=A_{j}^{u}+s^{u} \times Y_{j}^{u}
\end{gathered}
$$

$$
I_{j}^{u}=\sum_{v=u+1}^{N} \int_{t \in\left(D_{F(j)}^{u}, D_{j}^{u}\right]} \delta_{j}^{u, v} \times P^{u, v}(t) d t
$$

$$
O_{j}^{u}=\sum_{v=1}^{u-1} \int_{t \in\left(D_{F(j)}^{v}, D_{j}^{v}\right]} \delta_{j}^{v, u} \times P^{v, u}(t) d t
$$

$$
\begin{gathered}
X_{j}^{u}=X_{j}^{u-1}+I_{j}^{u}-O_{j}^{u} \\
T_{j}=\sum_{u=1}^{N-1} r^{u}+\sum_{u=1}^{N} Y_{j}^{u} \times s^{u}
\end{gathered}
$$

$$
D_{j}^{1} \geq \min _{j^{\prime}<j}\left\{D_{j^{\prime}}^{1}+T_{j^{\prime}}\right\} \quad(j=m+1, m+2, \cdots, K)
$$

Equation (1) shows the relation between parameters $\delta_{j}^{u, v}$ and $Y_{j}^{u}$. Equation (2) and equation (3) calculate the arrival and departure time respectively. Equation (4) and equation (5) compute the number of boarded and alighted passengers respectively. Equation (6) interprets the flow conservation. Equation (7) calculates the circulation time and inequality (8) ensures the departure time of a bus at the terminal after the allowable moment.

\subsection{Objective}

It is well known that the number of vehicles operate on a given transit line is limited. In the morning rush hours, all buses are put into operation for carrying passengers as many as possible and the maximum ticket income is achieved simultaneously. It is thus that the transit company has the same purpose with the passengers. A better schedule should provide a comfortable travel service during the stay of the passengers at the stations and in the vehicles. The service at the stations can be estimated by the waiting times and the service in the 
vehicles can be measured by the crowded costs. Such a well-designed schedule should match the timedependent demand and accelerate the circulative utilizations of vehicles.

In order to count the overall waiting times at the stations, this study introduce a parameter, $S(j)$, to index the earliest bus which is posterior to bus $j$ with $\delta_{j}^{u, v}=0$ and $\delta_{S(j)}^{u, v}=1 . S(j)$ can be achieved as follows.

$$
S(j)=\min \left\{j^{\prime} \mid j^{\prime}>j,\left(1-\delta_{j}^{u, v}\right) \times \delta_{j^{\prime}}^{u, v}=1\right\}
$$

The waiting times of passengers at station $u$ associated with bus $j$ is thus represented as below.

$$
\begin{aligned}
& \sum_{v=u+1}^{N} \int_{t \in\left(D_{F(j)}^{u}, D_{j}^{u}\right]} \delta_{j}^{u, v} \times P^{u, v}(t) \times\left(D_{j}^{u}-t\right) d t \\
& +\sum_{v=u+1}^{N} \int_{t \in\left(D_{F(j)}^{u}, D_{j}^{u}\right]}\left(1-\delta_{j}^{u, v}\right) \times P^{u, v}(t) \times\left(D_{S(j)}^{u}-t\right) d t
\end{aligned}
$$

where the first item counts the waiting times of passengers boarding the current bus, the second item counts the waiting times of passengers boarding the next stopping vehicle.

This study introduces the in-vehicle crowded cost to evaluate travel condition in the vehicles. The cost is incurred while the number of onboard passengers exceeds the maximum loading capacity of a bus. As a result, the in-vehicle crowded cost for bus $j$ departing from station $u$ is $X_{j}^{u} \times r^{u}$ if $X_{j}^{u}>C$. The total invehicle crowded costs are thus presented as follows.

$$
\sum_{j=1}^{K} \sum_{u=1}^{N-1} X_{j}^{u} \times r^{u} \times I\left(X_{j}^{u}-C\right)
$$

where $I(x)$ is the sign function which equals to 1 if $x>0$ and 0 otherwise.

On condition that the number of vehicles is limited, the objective of the newly established model integrated the schedule with the skip-stop operation is to minimize the overall waiting times of passengers at stations and the total in-vehicle crowded costs. Such considerations yield the following objective function.

$$
\begin{aligned}
& \min Z=\sum_{j=1}^{K} \sum_{u=1}^{N-1} \sum_{v=u+1}^{N} \int_{t \in\left(D_{F(j)}^{u}, D_{j}^{u}\right]} \delta_{j}^{u, v} \times P^{u, v}(t) \times\left(D_{j}^{u}-t\right) d t \\
& +\sum_{j=1}^{K} \sum_{u=1}^{N-1} \sum_{v=u+1}^{N} \int_{t \in\left(D_{F(j)}^{u}, D_{j}^{u}\right]}\left(1-\delta_{j}^{u, v}\right) \times P^{u, v}(t) \times\left(D_{S(j)}^{u}-t\right) d t
\end{aligned}
$$

$+\sum_{j=1}^{K} \sum_{u=1}^{N-1} X_{j}^{u} \times r^{u} \times I\left(X_{j}^{u}-C\right)$

\section{Solution Algorithm}

The proposed model is a nonlinear programming problem which associates the zero-one variables and the parameters are tightly related to each other. It is hardly solved with conventional solution methods. The genetic algorithm, ${ }^{13-15}$ which generates solutions to optimization problems using techniques inspired by natural evolution, is therefore adopted to solve the proposed model in this research.

\subsection{Bilevel genetic algorithm}

The transit skip-stop scheduling problem is actually to determine the departure times of vehicles at the terminal and the skip-stop operations on the line, which can be viewed as two stages. The first stage decides the departure times and the second one determines the skipstop strategy at the intermediate stations. According to such layered characteristics, the bilevel genetic algorithm, ${ }^{16,17}$ an improved procedure to solve the hierarchical optimization problem within a single framework, is developed to solve the proposed model. The possible departure times of vehicles at the terminal are searched by the outer genetic algorithm and the skip-stop operations are solved by the inner genetic algorithm. A brief outline of the bilevel genetic algorithm is given below.

\section{Begin}

generate the initial population randomly for the outer layer genetic algorithm

while the outer layer stopping criterion is not satisfied do

apply crossover operator

apply mutation operator

optimize fitness

generate the initial population randomly for the inner layer genetic algorithm

while the inner layer stopping criterion is not satisfied do

apply crossover operator

apply mutation operator

evaluate fitness

end 


\section{end \\ End}

\subsection{Coding approach}

A special binary coding approach has been proposed to solve the model. For the outer genetic algorithm, each gene location in a chromosome corresponds to a possible departure time of vehicles at the terminal during the operation period as shown in Fig. 4, where ' 1 ' in this figure indicates departure and ' 0 ' indicates cancellation at the corresponding moment. If the lengths of time-slice span and operation period are respectively set to be one minute and one hour, a chromosome associates with 60 possible start points. For example, a gene string $(1,0,1,1, \cdots, 1,0,1)$ denotes that the departure times of vehicles at the terminal are 7:00, 7:02, $7: 03, \cdots, 7: 57,7: 59$ respectively if the operation period is from 7:00 to 7:59.

The terminal

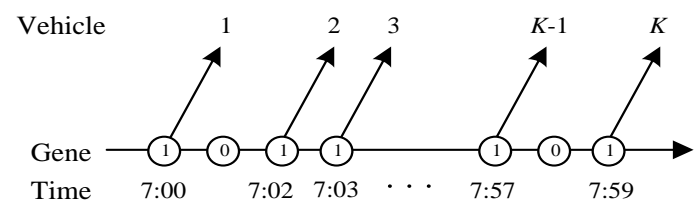

Fig. 4. The coding illustration of a chromosome for the outer genetic algorithm

For the inner layer genetic algorithm, each gene location in a chromosome represents a possible skip-stop choice while the buses traverse every station shown in Fig. 5, where ' 1 ' indicates stop and ' 0 ' indicates skip at the corresponding station. In Fig. 5, a chromosome is divided into $K$ independent parts. Each part indicates the corresponding operation choices for every vehicle.

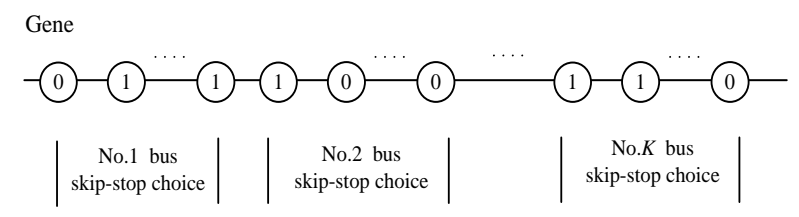

Fig. 5. The coding illustration of a chromosome for the inner genetic algorithm

\subsection{Genetic operators}

In view of the fact that the number of ' 0 ' is more than ' 1 ' in a chromosome on the outer genetic algorithm, this study builds a special mutation operator to respond this problem. The probability of ' 1 ' to ' 0 ' is taken a larger value in order to generate more ' 0 '. The other genetic operators of the algorithms are the same as the related references. ${ }^{18}$

\subsection{Fitness function}

The overall waiting time, the total in-vehicle crowded cost and the other parameters are estimated simultaneously by calculating the objective and equality constraints recursively from the first bus starting from the terminal step by step. However, the allowable departure time constraint referred in inequality (8) might not be feasible. In that case, a function is defined as Eq. (10) to obtain an unconstrained optimization problem that integrates the inequality constraint, and the fitness function is obtained by Eq. (11).

$$
\begin{gathered}
\bar{Z}=Z+\sum_{j=m+1}^{K} \beta_{j} \times \max \left\{0, \min _{j^{\prime}<j}\left\{D_{j^{\prime}}^{1}+T_{j^{\prime}}\right\}-D_{j}^{1}\right\} \\
\text { fitness }=\frac{\bar{Z}_{\max }-\bar{Z}}{\bar{Z}_{\max }-\bar{Z}_{\min }}
\end{gathered}
$$

where $Z$ is the objective from Eq. (9), $\bar{Z}_{\max }$ and $\bar{Z}_{\text {min }}$ denote the maximum and minimum values of parameter $\bar{Z}$ obtained from Eq. (10), and $\beta_{j}$ denotes penalty factors.

\section{Numerical Example}

The procedure introduced in this paper is applied to design the skip-stop scheduling for the No.34 bus line in Lanzhou City of China. The line takes a ring shape and associates with 15 stations. The operation time is from 7:00 to 8:00, the time-dependent demand patterns are counted in terms of one minute interval. The sure-stop and choice-stop sets are $\Phi_{1}=\{1,3,9,11,13,15\}$ and $\Phi_{2}=\{2,4,5,6,7,8,10,12,14\}$ respectively.

Each transit vehicle has a capacity of 60 passengers. The number of supplied vehicles is 15 and the dwell time of all stations is 0.7 minute. The running times between two adjacent stations are given in Table 1, and the parameter values for the bilevel genetic algorithm are listed in Table 2. 
Table 1 . The running times between two adjacent stations (min)

\begin{tabular}{cccc}
\hline Adjacent Stations & $\begin{array}{c}\text { Running } \\
\text { Time }\end{array}$ & Adjacent Stations & $\begin{array}{c}\text { Running } \\
\text { Time }\end{array}$ \\
\hline Lanzhou Rail Station-Hezheng Road & 2.0 & Municipal Party Committee -Xiguan Hub & 2.3 \\
Hezheng Road-Tianshui Intersection & 2.1 & Xiguan Hub-Shuangchengmen & 2.1 \\
Tianshui Intersection-Jincheng Hotel & 1.9 & Shuangchengmen-Nanguan Hub & 2.5 \\
Jincheng Hotel-Nanbinhe Road & 2.3 & Nanguan Hub-Zhongshanlin & 2.3 \\
Nanbinhe Road-Traffic Police Force & 2.4 & Zhongshanlin-Wuquan Square & 2.1 \\
Traffic Police Force-City Hall & 2.2 & Wuquan Square-Renhengguoji & 2.4 \\
City Hall-Municipal Party Committee & 2.4 & Renhengguoji-Lanzhou Rail Station & 1.8 \\
\hline
\end{tabular}

Table 2. Parameters list of the bilevel genetic algorithm

\begin{tabular}{llllll}
\hline Type & Parameter & Value & Type & Parameter & Value \\
\hline & Population size & 50 & & Population size & 100 \\
& Length of chromosome & 60 & & Length of chromosome & $9 \times K$ \\
\multirow{3}{*}{ Outer GA } & Number of generations & 400 & & Number of generations & 200 \\
& Probability of crossover & 0.90 & Inner GA & Probability of crossover & 0.90 \\
& Probability of mutation $(0 \rightarrow 1)$ & 0.15 & & Probability of mutation & 0.10 \\
& Probability of mutation $(1 \rightarrow 0)$ & 0.65 & & & \\
\hline
\end{tabular}

The optimal solution has been achieved after 238 iterations in the outer genetic algorithm as shown in Fig. 6 , which also illustrates the robustness and effectiveness of the algorithm. Using the obtained solution, the departure times of buses at the terminal and the skipstop operations along the intermediate stations are given in Table 3. The illustration of corresponding schedule is shown in Fig. 7.

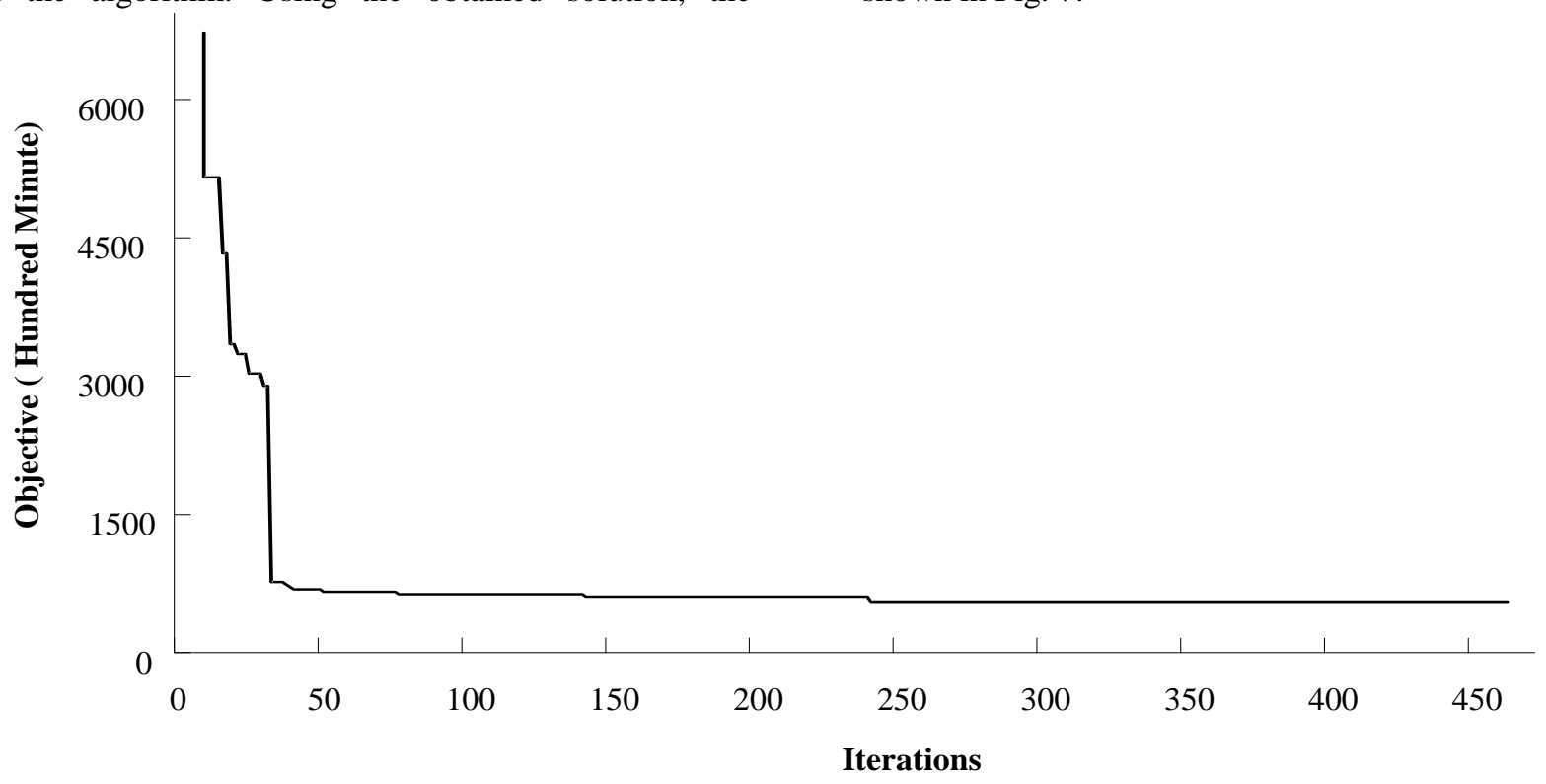

Fig. 6. The variation curve on the objective with iterations

Table 3. The optimal departure times at the terminal and skip-stop operation

\begin{tabular}{cccccccccccccccccc}
\hline Bus & \multicolumn{1}{c}{} & \multicolumn{1}{c}{ Departure Time and Skip-Stop Operation } & \multicolumn{1}{c}{ Supplied } & Load-Rate \\
Run & 1 & 2 & 3 & 4 & 5 & 6 & 7 & 8 & 9 & 10 & 11 & 12 & 13 & 14 & 15 & Vehicle & $(\%)$ \\
\hline 1 & $7: 00$ & 1 & 1 & 1 & 1 & 1 & 1 & 0 & 1 & 1 & 1 & 0 & 1 & 1 & 1 & 1 & 124.17 \\
2 & $7: 02$ & 1 & 1 & 1 & 1 & 0 & 0 & 1 & 1 & 0 & 1 & 1 & 1 & 0 & 1 & 2 & 83.33 \\
\hline
\end{tabular}


Table 3 (Continued). The optimal departure times at the terminal and skip-stop operation

\begin{tabular}{cccccccccccccccccccc}
\hline 3 & $7: 04$ & 1 & 1 & 0 & 1 & 1 & 0 & 1 & 1 & 1 & 1 & 1 & 1 & 0 & 1 & 3 & 89.44 \\
4 & $7: 08$ & 0 & 1 & 0 & 1 & 1 & 0 & 0 & 1 & 1 & 1 & 1 & 1 & 0 & 1 & 4 & 91.11 \\
5 & $7: 12$ & 0 & 1 & 0 & 0 & 0 & 1 & 1 & 1 & 0 & 1 & 1 & 1 & 0 & 1 & 5 & 110.00 \\
6 & $7: 14$ & 1 & 1 & 0 & 1 & 0 & 0 & 0 & 1 & 1 & 1 & 0 & 1 & 1 & 1 & 6 & 77.22 \\
7 & $7: 17$ & 0 & 1 & 0 & 0 & 0 & 1 & 1 & 1 & 0 & 1 & 0 & 1 & 1 & 1 & 7 & 72.78 \\
8 & $7: 21$ & 1 & 1 & 0 & 0 & 1 & 0 & 0 & 1 & 1 & 1 & 1 & 1 & 1 & 1 & 8 & 104.17 \\
9 & $7: 24$ & 0 & 1 & 0 & 1 & 1 & 0 & 1 & 1 & 1 & 1 & 1 & 1 & 0 & 1 & 9 & 87.78 \\
10 & $7: 28$ & 1 & 1 & 1 & 0 & 0 & 0 & 0 & 1 & 1 & 1 & 0 & 1 & 0 & 1 & & 10 & 108.33 \\
11 & $7: 32$ & 0 & 1 & 1 & 1 & 0 & 0 & 1 & 1 & 0 & 1 & 0 & 1 & 1 & 1 & & 11 & 86.11 \\
12 & $7: 36$ & 0 & 1 & 1 & 0 & 0 & 0 & 0 & 1 & 0 & 1 & 1 & 1 & 0 & 1 & 12 & 81.39 \\
13 & $7: 39$ & 1 & 1 & 0 & 0 & 0 & 1 & 0 & 1 & 1 & 1 & 0 & 1 & 0 & 1 & 13 & 90.00 \\
14 & $7: 43$ & 0 & 1 & 0 & 1 & 1 & 0 & 1 & 1 & 1 & 1 & 0 & 1 & 0 & 1 & 14 & 88.89 \\
15 & $7: 44$ & 0 & 1 & 1 & 1 & 0 & 0 & 1 & 1 & 0 & 1 & 0 & 1 & 0 & 1 & 15 & 71.94 \\
16 & $7: 45$ & 1 & 1 & 0 & 1 & 1 & 1 & 0 & 1 & 1 & 1 & 0 & 1 & 1 & 1 & 1 & 70.28 \\
17 & $7: 46$ & 1 & 1 & 1 & 0 & 1 & 1 & 1 & 1 & 0 & 1 & 1 & 1 & 1 & 1 & & 2 & 82.50 \\
18 & $7: 48$ & 0 & 1 & 0 & 1 & 1 & 0 & 0 & 1 & 0 & 1 & 0 & 1 & 1 & 1 & 3 & 62.50 \\
19 & $7: 49$ & 1 & 1 & 0 & 0 & 0 & 0 & 1 & 1 & 0 & 1 & 1 & 1 & 1 & 1 & 4 & 74.44 \\
20 & $7: 51$ & 1 & 1 & 1 & 1 & 1 & 1 & 1 & 1 & 1 & 1 & 0 & 1 & 0 & 1 & 5 & 93.06 \\
21 & $7: 53$ & 0 & 1 & 0 & 1 & 0 & 1 & 1 & 1 & 0 & 1 & 1 & 1 & 0 & 1 & 6 & 76.11 \\
22 & $7: 55$ & 1 & 1 & 0 & 1 & 0 & 1 & 1 & 1 & 0 & 1 & 1 & 1 & 0 & 1 & & 97.50 \\
23 & $7: 57$ & 1 & 1 & 0 & 0 & 1 & 1 & 0 & 1 & 1 & 1 & 0 & 1 & 0 & 1 & 8 & 83.33 \\
24 & $7: 59$ & 1 & 1 & 1 & 1 & 1 & 0 & 1 & 1 & 1 & 1 & 1 & 1 & 0 & 1 & 9 & 115.83 \\
\hline
\end{tabular}

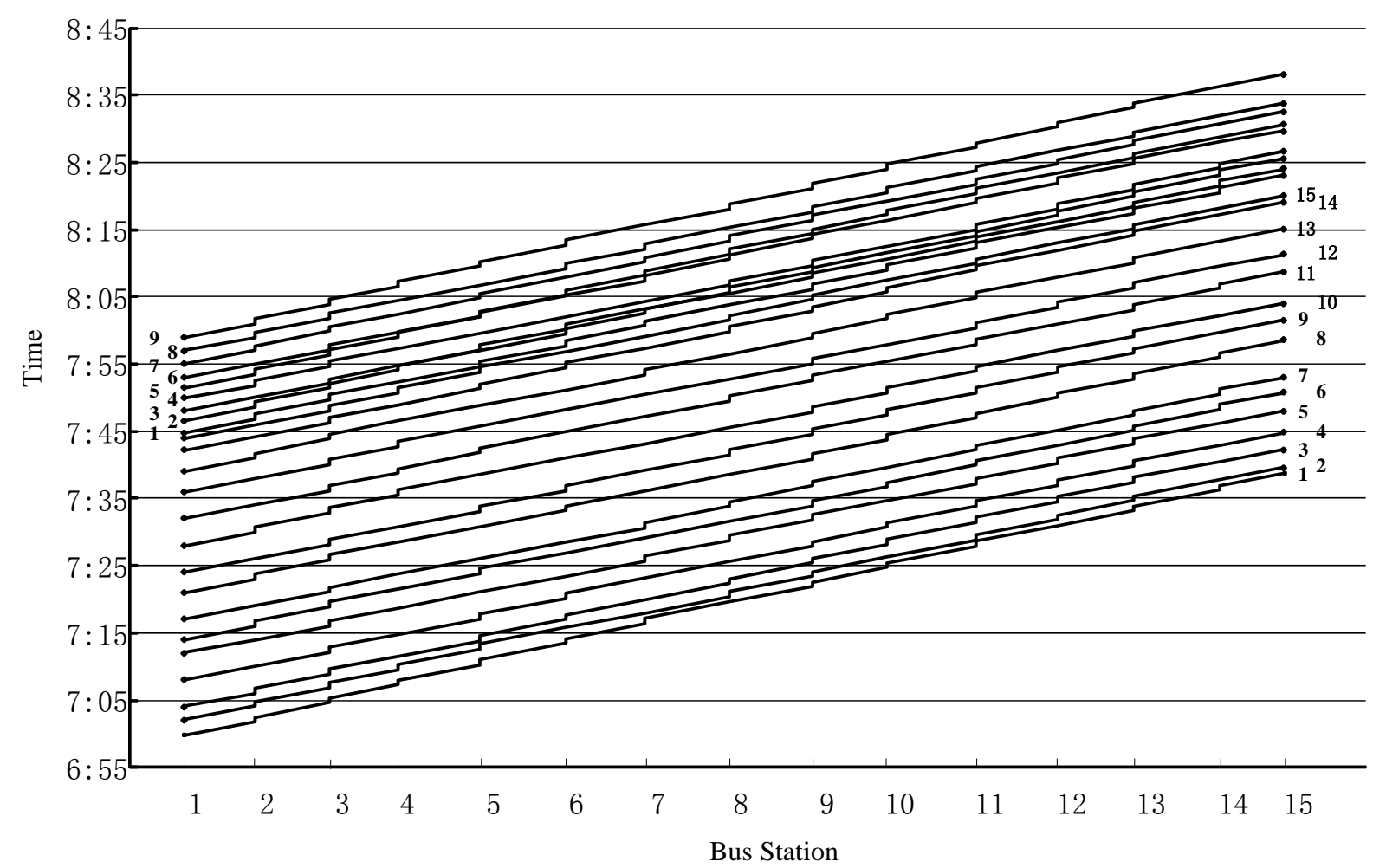

Fig. 7. The illustration of corresponding transit schedule 
Table 4. The regular schedule with even interval and all-stop operation

\begin{tabular}{cccccc}
\hline Bus Number & Departure Time & Supplied Vehicle & Bus Number & Departure Time & Supplied Vehicle \\
\hline 1 & $7: 00: 00$ & 1 & 12 & $7: 32: 27$ & 12 \\
2 & $7: 02: 57$ & 2 & 13 & $7: 35: 24$ & 13 \\
3 & $7: 05: 54$ & 3 & 14 & $7: 38: 21$ & 14 \\
4 & $7: 08: 51$ & 4 & 15 & $7: 41: 18$ & 15 \\
5 & $7: 11: 48$ & 5 & 16 & $7: 44: 15$ & 2 \\
6 & $7: 14: 45$ & 6 & 17 & $7: 47: 12$ & 3 \\
7 & $7: 17: 42$ & 7 & 18 & $7: 50: 09$ & 4 \\
8 & $7: 20: 39$ & 8 & 19 & $7: 53: 06$ & 5 \\
10 & $7: 23: 36$ & 9 & 20 & $7: 56: 03$ & 6 \\
\end{tabular}

Table 5. The comparison between two transit scheduling schemes

\begin{tabular}{ccccccc}
\hline Schedule Plan & $\begin{array}{c}\text { Number of } \\
\text { Departed Buses }\end{array}$ & $\begin{array}{c}\text { Objective } \\
(\mathrm{min})\end{array}$ & $\begin{array}{c}\text { Average } \\
\text { Waiting } \\
\text { Time(min) }\end{array}$ & $\begin{array}{c}\text { Average Load- } \\
\text { Rate(\%) }\end{array}$ & $\begin{array}{c}\text { Crowed } \\
\text { Cost(min) }\end{array}$ & $\begin{array}{c}\text { Waiting } \\
\text { Time(min) }\end{array}$ \\
\hline $\begin{array}{c}\text { Designed Plan } \\
\text { Regular Plan }\end{array}$ & 24 & 17379.18 & 2.33 & 88.43 & 11905.00 & 5474.18 \\
\hline
\end{tabular}

In order to explain comparatively the advantage of the proposed transit scheduling, the regular scheduling with the same supplied vehicles and other parameters are obtained by a simple computation, as shown in Table 4 . The comparison of the effect between the optimal schedule designed in this study and the corresponding regular schedule are given in Table 5.

It is found in Table 5 that there are 24 buses departing from the terminal by the constructed schedule. However, only 21 runs are achieved by the regular schedule as the inadequate circulation time. In contrast, the proposed schedule evidently reduces the waiting time and the invehicle crowed level, which results in the improvement of transit service quality.

\section{Conclusions}

Based on the analysis of the passenger demand and the bus operation process in a transit system, this paper studies the problem of transit scheduling with the skipstop operation for a congested bus line. A nonlinear optimization model with the objective of minimizing the total of passenger waiting time and the in-vehicle crowded costs is established. The resource constraint associated with available fleet has been taken into a full consideration in this study. A hybrid procedure embedded in bilevel genetic algorithm is used to solve the proposed model. The validation of the model and the algorithm has been proved with the help of a real-world case. It should be noted that the running time of a bus is assumed to be a constant which, as a matter of fact, may be random or fuzzy under the influence of traffic signals, road condition, and driving psychology. Therefore, it is necessary to treat the transit scheduling problem under a random or fuzzy environment. Moreover, the intense competition among transit supplies also makes it urgent to further study the transit scheduling problem because some passengers may select other transit modes when they are facing heavy congestions. 


\section{Acknowledgements}

This research work was supported in part by National Natural Science Foundation of China (No. 50968009) and Research Fund for the Doctoral Program of Higher Education of China (No. 20096204110003).

\section{References}

1. J.H.Jansson, A simple bus line model for optimization of service frequency and bus size, Journal of Transport Economic and Policy, 14(1) (1980) 53-80.

2. A. Ceder, Bus timetables with even passenger loads as opposed to even headways, Transportation Research Record, 1760 (2001) 3-9.

3. S. C. Wirasinghe, Initial planning for urban transit systems, Advanced Modeling for Transit Operations and Service Planning, Elsevier Science, Oxford, (2003) $1-29$.

4. V. Guihaire, J. K. Hao, Transit network design and scheduling: a global review, Transportation Research Part A, 42 (10) (2008) 1251-1273.

5. P. Prioni, D. Hensher, Measuring service quality in scheduled bus services, Journal of Public Transportation, 3 (2000) 51-74.

6. J. H. Banks, Optimal headways for multi-route transit systems, Journal of Advanced Transportation, 24 (1990) 127-154.

7. K. K. T. Lee, S. H. F. Kuo, P. M. Schonfeld, Optimal mixed bus fleet for urban operations, Transportation Research Record, 1503 (1995) 39-48.

8. T. A. S. Vijayaraghavan, K. M. Anantharamaiah, Fleet assignment strategies in urban transportation using express and partial services, Transportation Research Part A, 29(2) (1995) 157-171.

9. S. P. Delle, F. Filippi, Service optimization for bus corridors with short-turn strategies and variable vehicle size, Transportation Research Part A, 32(1) (1998) 19-38.

10. X.J. Eberlein, N. Wilson, B. Bernstein, The real time deadheading problem in transit operations control, Transportation Research Part B, 32(2) (1998) 77-109.

11. L. Fu, Q. Liu, P. Calamai, A real-time optimization model for dynamic scheduling of transit operations. Transportation Research Record, 1857 (2003) 48-55.

12. C. Leiva, J. C. Muzoa, R. Giesen, H. Larrain, Design of limited-stop services for an urban bus corridor with capacity constraints, Transportation Research Part B, 44(10) (2010) 1186-1201.

13. H. M. Niu, A. Z. Hu, Optimization model and algorithm for system operation division of labor at two-way marshalling station, Science in China Part E, 41 (5) (1998) 511-518.

14. J. Gao, R. Chen, A hybrid genetic algorithm for the distributed permutation flowshop scheduling problem, International Journal of Computational Intelligence
Systems, 4( 4) (2011) 497-508.

15. R.S. Kumar, N. Alagumurthi, Integrated total cost and tolerance optimization with genetic algorithm, International Journal of Computational Intelligence Systems, 3(3) (2010) 325-333.

16. Y. Marinakisa, M. Marinakib, A bilevel genetic algorithm for a real life location routing problem, International Journal of Logistics Research and Applications, 11(1) (2008) 49-65.

17. Z. X. Guo, W. K. Wong, S. Y. S. Leung, J. T. Fan, S. F. Chan, A bi-level genetic algorithm for multi-objective scheduling of multi-and mixed-model apparel assembly lines, Lecture Notes in Computer Science, 4304 (2006) 934-941.

18. M. Gen, R. W. Cheng, Genetic Algorithms and Engineering Optimization (John Wiley \& Son, 2000). 